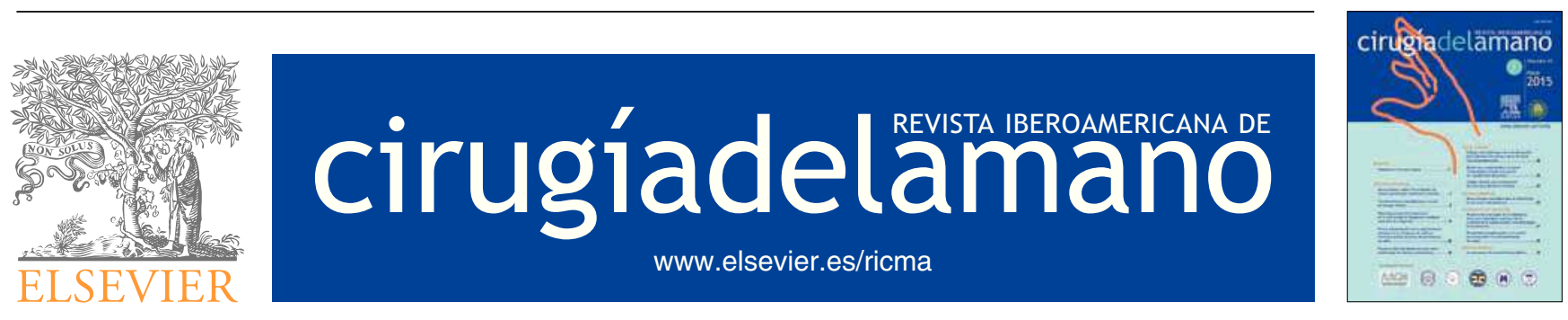

TÉCNICA QUIRÚRGICA

\title{
Nueva técnica supinadora para la deformidad en pronación del antebrazo
}

\author{
J.M. Rotella, P. Rotella*, A. Escobedo, J.M. Herrera, M. Rotella y C.I. Rotella
}

Servicio de Cirugía Ortopédica y Traumatología, Sanatorio del Norte, San Miguel de Tucumán, Argentina

Recibido el 15 de febrero de 2015. Aceptado el 15 de marzo de 2015.

\section{PALABRAS CLAVE \\ Transferencias tendinosas; Deformidades mano; Espasticidad muscular}

\section{KEYWORDS}

Tendon transfer; Hand deformities; Muscle spasticity

\begin{abstract}
Resumen
El síndrome espático es una encefalopatía no progresiva con alteraciones motoras, sensitivas e intelectuales. La deformidad en pronación afecta el uso de la extremidad al coger y manipular objetos. El tener la mano en pronación y no poder llevar objetos a la vista influye negativamente en el aprendizaje.

En este trabajo se presenta una nueva técnica supinadora en la que se transfiere el pronador redondo a un hemitendón del braquiorradial que mantiene su inserción en el radio.

La indicación para su realización es un paciente con hemiparesia espástica, con el antebrazo en pronación marcada, muñeca en extensión y pulgar en flexión y aducción. Es imprescindible que el paciente mantenga una aceptable función de prensión y no presente contracturas ni rigideces articulares.
\end{abstract}

( $)$ 2015, SECMA. Publicado por Elsevier España, S.L.U. Este es un artículo Open Acces distribuido bajo los términos de la licencia CC BY-NC-ND (http://creativecommons.org/licenses/by-nc-nd/4.0/).

New supination technique for forearm pronation deformity

\begin{abstract}
The spastic syndrome is a non-progressive encephalopathy with motor, sensory, and intellectual disorders. The deformity in pronation affects the use of the upper extremity when holding and manipulating objects. Having the hand in pronation and the inability to hold objects to the view has a negative influence on learning.

A new technique for supination is presented in this article. The pronator teres is transferred to a hemi-tendon of the brachioradialis tendon, which maintain its insertion in the radius.

The indication for this technique is a patient with spastic hemiparesis, with a forearm in marked pronation, extended wrist and thumb in flexion and adduction. A good pressing function and the absence of joint stiffnes are essential.

(c) 2015, SECMA. Published by Elsevier España, S.L.U. This is an open access article under the CC BY-NC-ND license (http://creativecommons.org/licenses/by-nc-nd/4.0/).
\end{abstract}

*Autor para correspondencia.

Correo electrónico: pablorotella@hotmail.com (P. Rotella).

( ) 2015, SECMA. Publicado por Elsevier España, S.L.U. Este es un artículo Open Acces distribuido bajo los términos de la licencia CC BY-NC-ND (http://creativecommons.org/licenses/by-nc-nd/4.0/). 


\section{Introducción}

El "síndrome espástico" se define como una encefalopatía no progresiva con alteraciones de las funciones motoras, sensitivas e intelectuales ${ }^{1-3}$.

Entre los parámetros que se deben tener en cuenta, están los diversos estadios madurativos del niño, especialmente el nivel de uso de la mano. Si el niño ignora totalmente la mano, se debe en parte al deterioro intelectual, pero también al déficit importante de sensibilidad. Al no tener "sensaciones" no incluye la mano como un instrumento de uso que le permita adquirir conocimiento y mejorar su nivel intelectual. También es importante tener en cuenta el desarrollo de la dominancia, dado que no es habitual que el niño la desarrolle antes de los tres años.

La deformidad en pronación del antebrazo, también afecta significativamente el uso de la extremidad al coger y manipular objetos. La palma de la mano no puede colocarse enfrentada con la otra mano, por lo que se dificulta la manipulación de los objetos. Cuando la deformidad en pronación es pronunciada, el niño realiza el apoyo de objetos con el borde radial de la mano y muñeca, y cuando es severa, realiza la postura de "prensión invertida", realizando la prensión con el borde cubital de mano.
El no poder llevar la mano en supinación evita el poder mostrar los objetos a la vista y por tanto se influye negativamente en el aprendizaje, puesto que cada vez se utiliza menos la mano y entra en un círculo de "falta de uso aprendido".

En los pacientes con disfunción cerebral la sensibilidad epicrítica es normal (dolor, frío y calor) por lo que la reeducación sensitiva se debe centrar en este tipo de sensibilidad y favorecer el desarrollo de otras sensaciones (tacto perioral), o la utilización de otros sentidos (como la vista) para desarrollar "nuevos engramas cerebrales de aprendizaje" que le permitan salir de este "círculo de deterioro intelectual": limitaciones motoras, limitaciones sensitivas y limitaciones intelectuales.

Todas las clasificaciones se centran en las deformidades, etiología y capacidad funcional motora ${ }^{2,4-7}$. Se pueden diferenciar dos tipos de técnicas quirúrgicas en esta patología. Las que intentan "mejorar la deformidad en pronación", como es la realización de tenotomías, liberación proximal del pronador ${ }^{8}$, toxina botulínica o técnicas para conseguir la relajación muscular y las que intentan "producir supinación" como son las transferencias tendinosas.

Para la realización de las transferencias tendinosas, es clásico la elección del pronador redondo, para lograr la extensión de la muñeca y a su vez disminuir su acción prona-
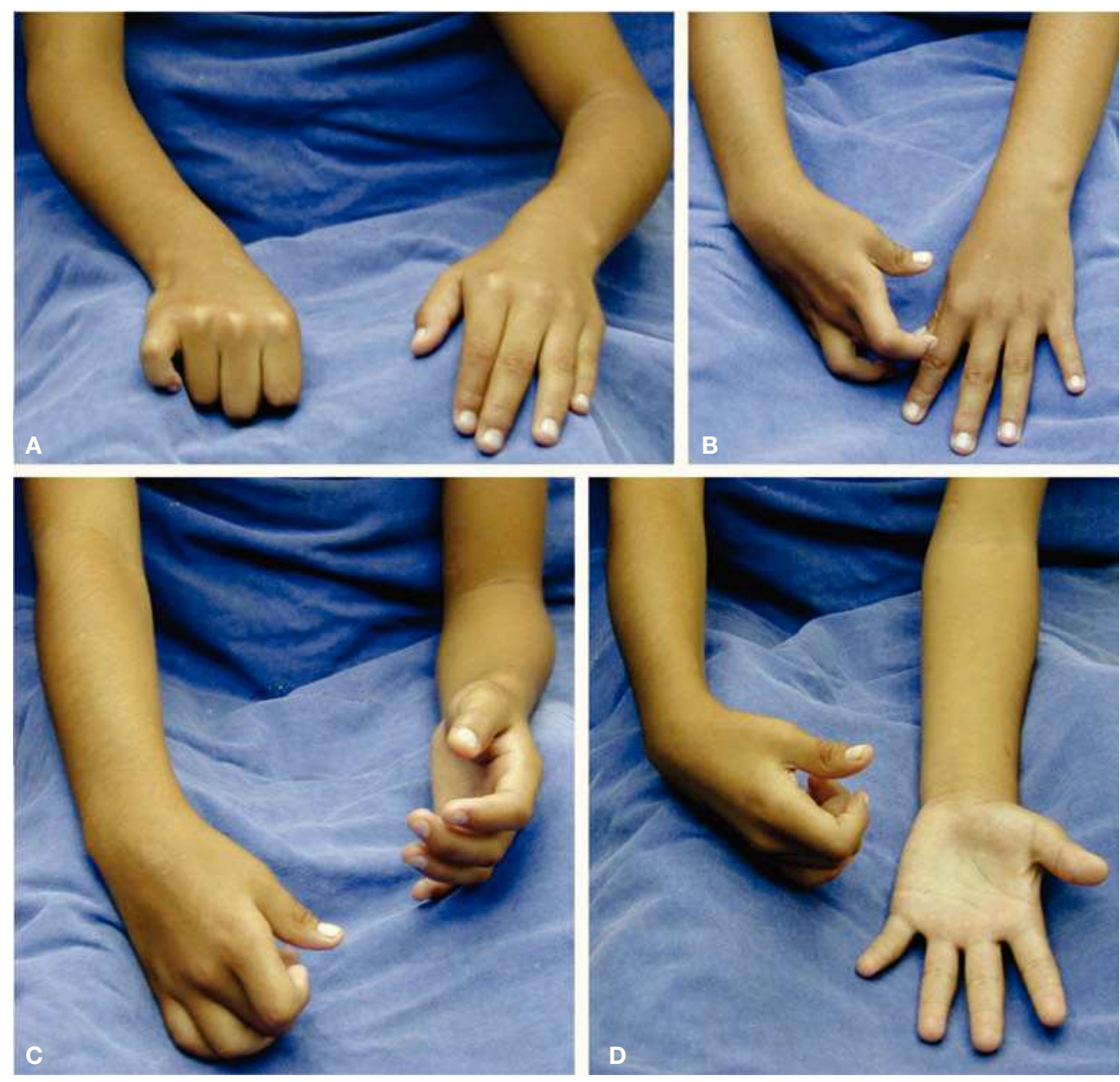

Figura 1 (A) Deformidad en pronación del antebrazo. (B) Para lograr la apertura del pulgar, el paciente precisa mantener la muñeca en flexión. (C y D) Supinación activa del miembro afecto comparada con el contralateral. 
dora y mejorar la supinación. Esta transferencia se puede realizar a través de la membrana interósea, para reinsertarlo en la zona volar del radio'.

También se ha descrito la transferencia del tendón del cubital anterior, por el lado cubital de la muñeca, lo que generar una fuerza que mejora la supinación ${ }^{10,11}$. Esta transferencia se puede realizar hacia el radio a nivel de la inserción del braquiorradial, a los extensores del carpo o al extensor común de los dedos ${ }^{12-14}$. El problema de esta última transferencia es que, la supinación del antebrazo con los dedos extendidos, puede ayudar en la higiene, pero no ayuda en el aprendizaje, ya que para "ver un objeto" se necesita que la mano lo capture flexionando los dedos y lo ofrezca a la cara.

En cualquier caso, cada técnica supinadora tiene una indicación precisa, dependiendo tanto del grado de deformidad en pronación, como de los grados de supinación a obtener. Además, el número y fuerza de músculos supinadores activos y funcionantes también condicionarán la técnica a realizar.

En el presente trabajo, se detalla una técnica quirúrgica de transferencia del pronador redondo al braquiorradial, con la que se mejora la supinación del antebrazo al desinsertar el pronador redondo del radio y reorientarlo a la estiloides radial mediante su sutura con un hemitendón del braquiorradial.

\section{Indicaciones y contraindicaciones}

Como ya se ha mencionado, mejorando cierto grado de supinación, se consigue una mejor visión de los objetos y se ayudará a mejorar las condiciones de aprendizaje junto con la capacidad de higiene y alimentación.

Mejorar esta deformidad "precozmente" es importante, ya que esperar una recuperación espontánea, sería retrasar el aprendizaje del niño. Por ello, la edad para realizar la intervención quirúrgica es antes de los 5 años de edad.

La indicación de esta técnica quirúrgica son los pacientes con parálisis cerebral, que presentan una deformidad en pronación con flexión de muñeca, que tienen un pulgar en la palma, pero con aceptable acción motora de prensión y con una supinación pasiva que llegue a $0^{\circ}$ (neutro) (fig. 1).

El momento de realización es alrededor de los cinco años, pero en pacientes con marcada actitud en pronación, déficit absoluto de supinación y con problemas en la visión, se puede intervenir más precozmente.

La contraindicación fundamental son los pacientes sin capacidad de prensión de la mano (que no podrán coger o soltar objetos a pesar de mejorar la supinación), con rigideces articulares o retracciones musculares estructuradas que eviten una supinación pasiva completa.

\section{Anatomía quirúrgica}

La técnica quirúrgica consiste en la transferencia del tendón del pronador redondo a un hemitendón del braquiorradial (fig. 2).

El objetivo de la intervención es prolongar la tracción del músculo pronador redondo hacia distal, al borde radial de la

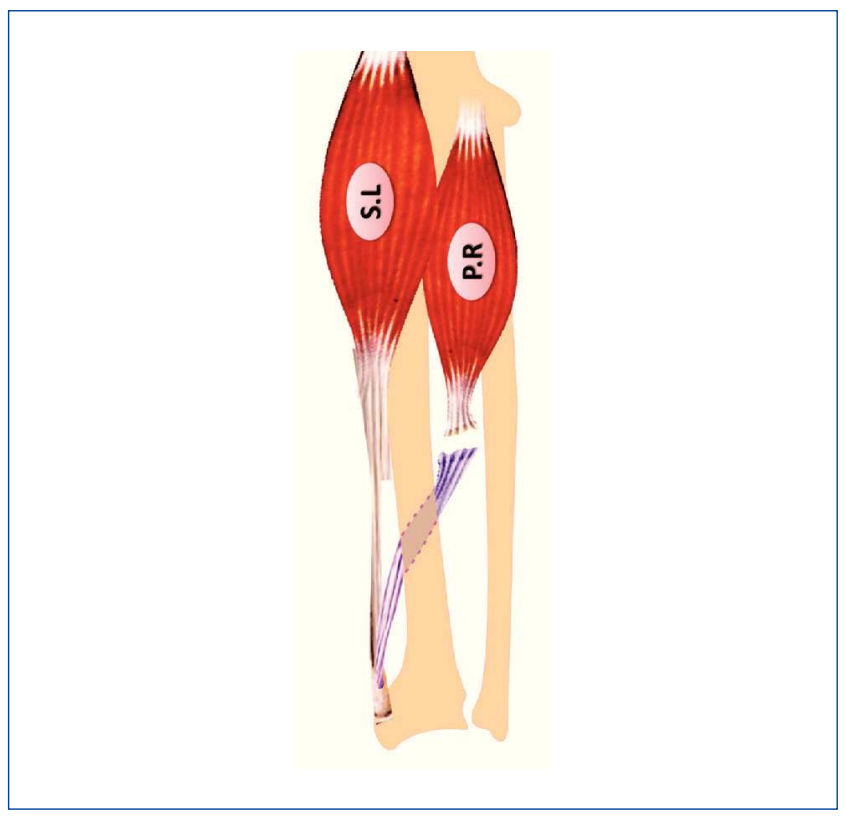

Figura 2 Transferencia del músculo pronador redondo a un hemitendón del braquiorradial.

base de la estiloides radial a través de la inserción del braquiorradial. Esta transferencia consigue que la contracción del pronador redondo produzca un movimiento de supinación del antebrazo.

Las estructuras en riesgo durante la intervención son la rama sensitiva del nervio radial, que se localiza bajo el músculo braquiorradial y la arteria radial durante el abordaje quirúrgico y el paquete vasculonervioso interóseo anterior durante el paso de la transferencia tendinosa por la membrana interósea.

Una de las ventajas de la técnica es que no precisa la inserción ósea de la transferencia, ya que se mantiene íntegra la inserción del tendón del músculo braquiorradial en la estiloides radial.

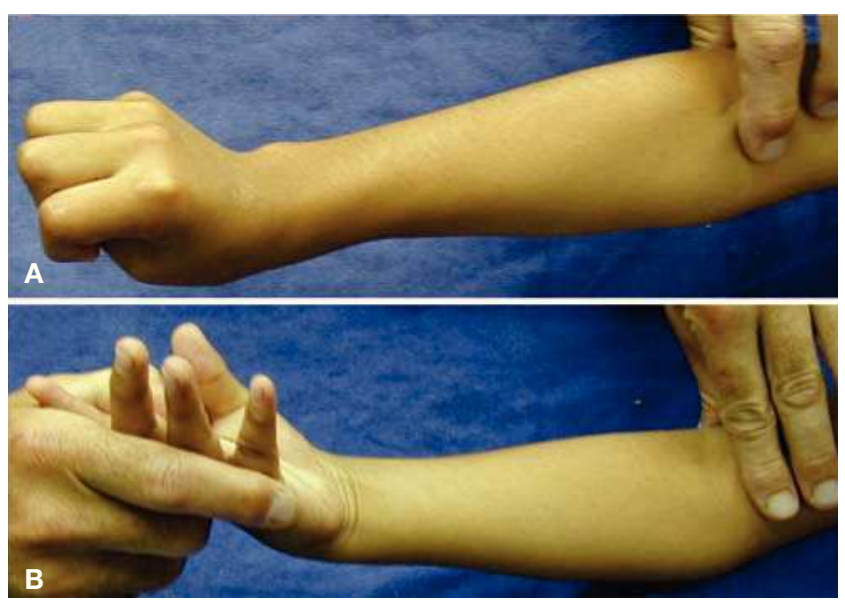

Figura 3 (A) Paciente con hemiparesia espástica. Antebrazo en pronación marcada, muñeca en extensión y pulgar en flexo y aducto. (B) Maniobra preoperatoria para comprobar la supinación pasiva completa del antebrazo. 
Previamente a la realización de la transferencia se debe evaluar las rigideces articulares tanto del codo como de la muñeca y las retracciones musculares, ya que su presencia contraindicaría la intervención.

El máximo pronador del antebrazo es el músculo pronador cuadrado, ayudado por el pronador redondo. El máximo supinador es el braquiorradial, ayudado por el bíceps braquial. Para evaluar la deformidad en pronación del antebrazo, se mantiene el codo a $90^{\circ}$ de flexión y se realiza una maniobra suave pero sostenida para relajar la espasticidad del pronador redondo. Se evalúa entonces el estado del pronador cuadrado (su retracción y espasticidad). Una vez lograda la supinación pasiva completa, se evalúa si el paciente es capaz de mantener esta posición (fig. 3).

\section{Técnica quirúrgica}

La técnica se realiza a través de una única incisión longitudinal de $3 \mathrm{~cm}$ en borde radial del tercio medio de antebrazo, a la altura de la inserción del pronador redondo (fig. 4A).

A continuación se incide la fascia entre la musculatura del braquiorradial y del flexor radial del carpo. Se separan ambos vientres musculares y se llega a la inserción del músculo pronador en el radio. Se debe tener especial precaución para no lesionar la arteria radial y la rama superficial del nervio radial que discurren justo bajo el músculo braquiorradial (fig. 5).
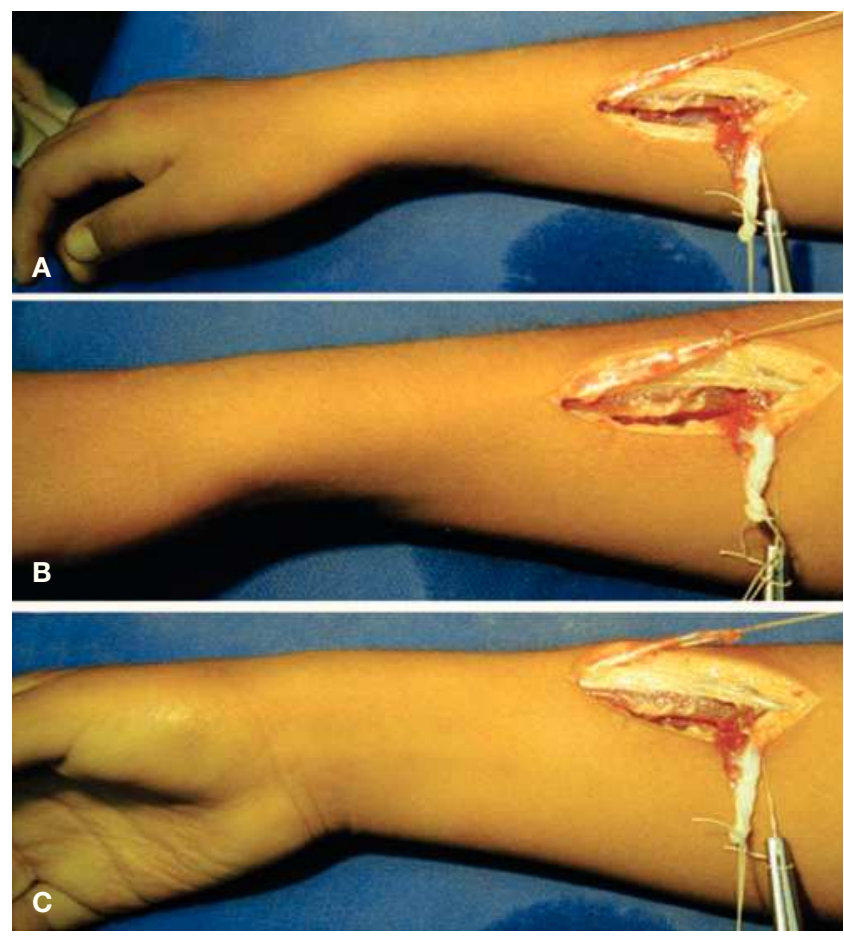

Figura 4 (A) Incisión en tercio medio del antebrazo se obtiene un hemitendón del braquiorradial y se desinserta el pronador redondo del radio. (B-C) Al traccionar del hemitendón del braquiorradial se consigue la supinación de la muñeca.
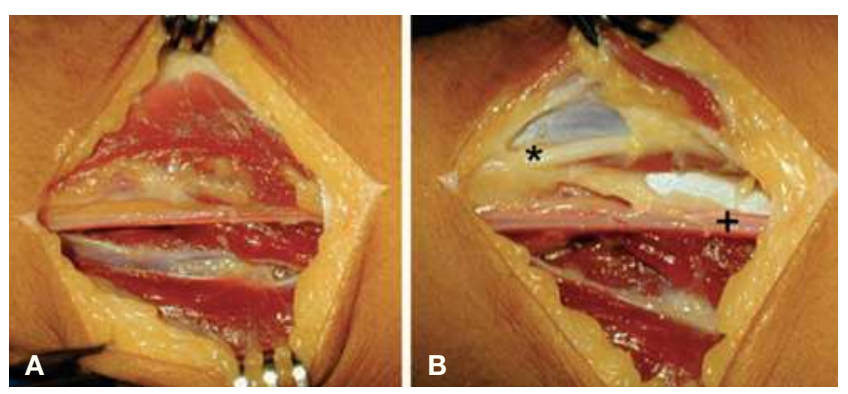

Figura 5 (A) Abordaje quirúrgico. (B) Identificación de arteria radial (signo suma) y del nervio radial (asterisco).

Se expone entonces la inserción del pronador redondo en el radio y se reseca toda su inserción, respetando la inserción más proximal, para no lesionar su inervación (fig. 6).

En la porción más distal de la incisión se expone el tendón del braquiorradial y se obtiene un hemitendón desde la unión musculo-tendinosa hasta su porción más distal, manteniendo su inserción en el radio. Se debe obtener una longitud suficiente que permita la sutura cómoda en la cara volar del antebrazo con el tendón del pronador redondo (fig. 7).

Una vez referenciados el tendón del pronador redondo y el hemitendón del braquiorradial, se realiza una ventana en la membrana interósea. Para ello, se debe disecar y retraer cuidadosamente el paquete vasculonervioso interóseo anterior. La incisión sobre la membrana interósea debe ser longitudinal, de $2 \mathrm{~cm}$ de largo y se debe tener precaución en respetar la inserción ósea de la membrana, ya que lesionarla podría condicionar la aparición de adherencias entre la transferencia tendinosa y la diáfisis del radio o del cúbito (fig. 8).

Para realizar el paso del hemitendón de dorsal a volar, se usa un hilo de tracción anudado al hemitendón del braquiorradial, que se pasa de dorsal a volar, a través de la ventana de la membrana interósea (fig. 9).

Por último, se realiza una sutura del hemitendón del braquiorradial al tendón del pronador redondo. Esta se realiza con el tendón del pronador redondo en máxima tensión y el antebrazo en supinación forzada. Para obtener una sutura resistente se recomienda que la sutura sea tipo Pulvertaft (fig. 10).

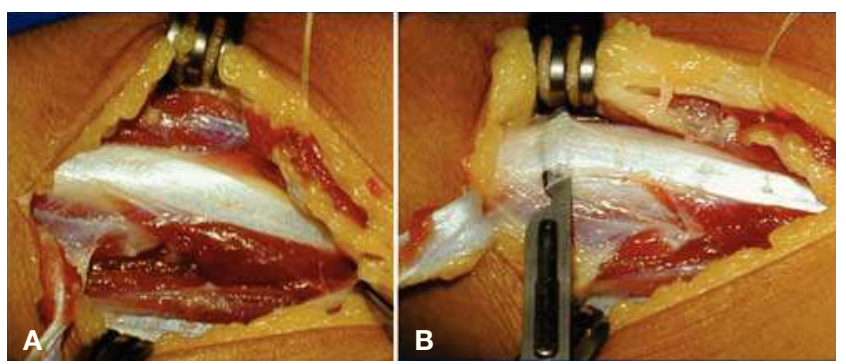

Figura 6 (A) Identificación de la inserción del músculo pronador redondo en el radio. (B) Desinserción del pronador del radio. 

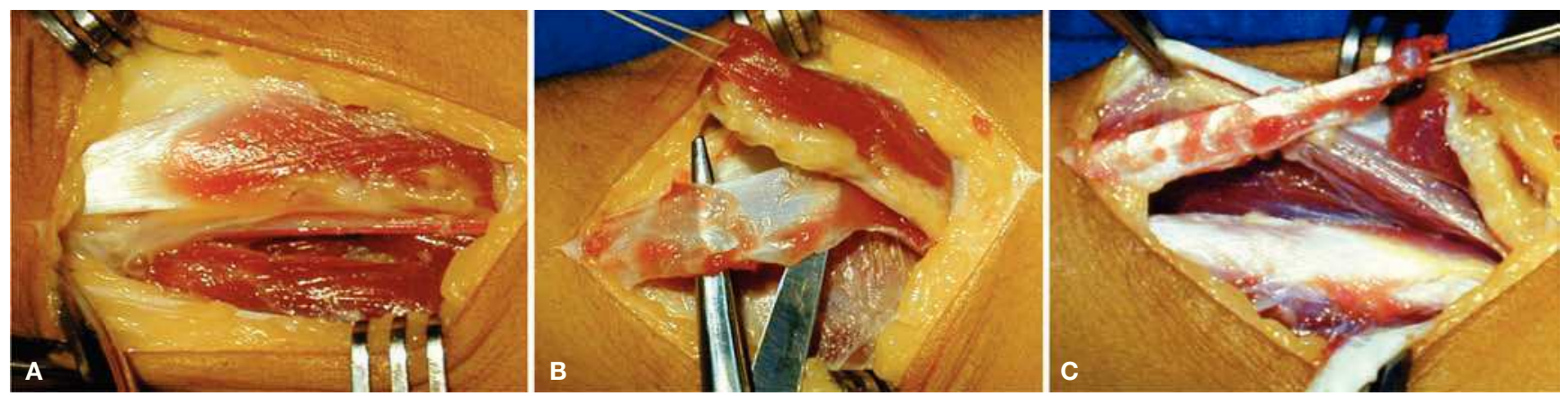

Figura 7 (A) Identificación tendón del braquiorradial. (B y C) Extracción del hemitendón del braquiorradial. Se utilizara la mitad más volar de su inserción para mejorar la acción supinadora.
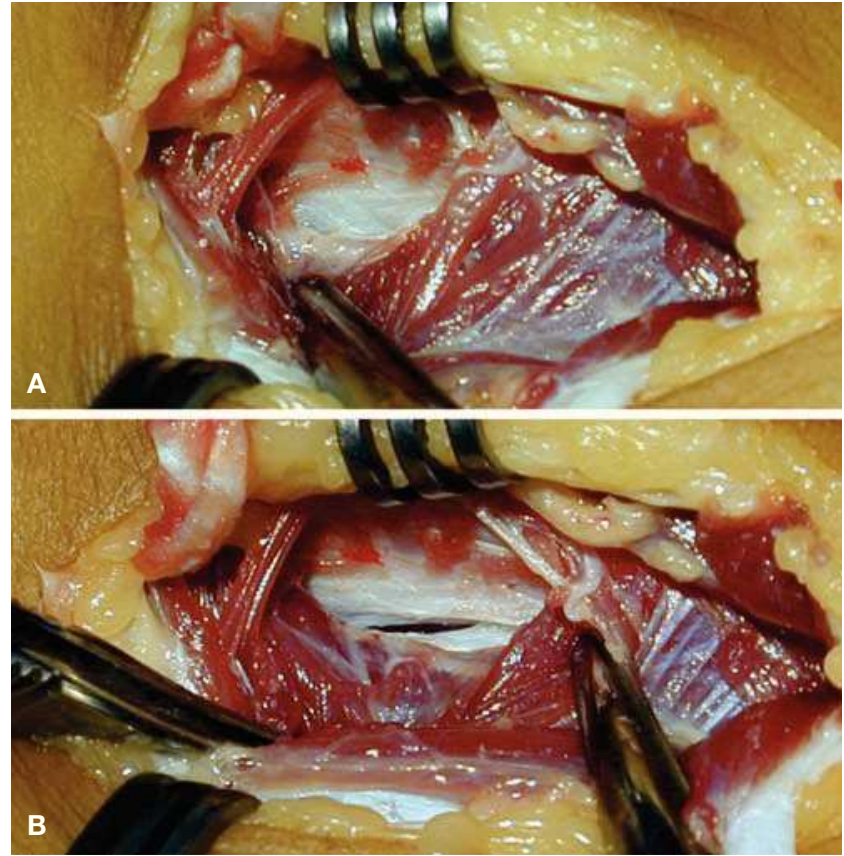

Figura 8 (A) Exposición de la membrana interósea, se retrae el vientre muscular de los flexores de los dedos y se protege el paquete neurovascular interóseo anterior. (B) Apertura de una "ventana" en el centro de la membrana interósea.

\section{Postoperatorio}

Al finalizar la intervención quirúrgica se coloca un yeso braquiopalmar, incluyendo todos los dedos. El codo debe mantenerse a $90^{\circ}$ de flexión, el antebrazo en supinación máxima, la muñeca en $70^{\circ}$ de extensión y los dedos en extensión completa durante un periodo de 6 semanas.

A las 6 semanas se retira el yeso y se comienza la rehabilitación, manteniendo una ortesis nocturna en supinación por un periodo de 6 meses más.

\section{Complicaciones}

Dentro de las complicaciones, la más frecuente es la neuropatía transitoria de la rama sensitiva del nervio radial,
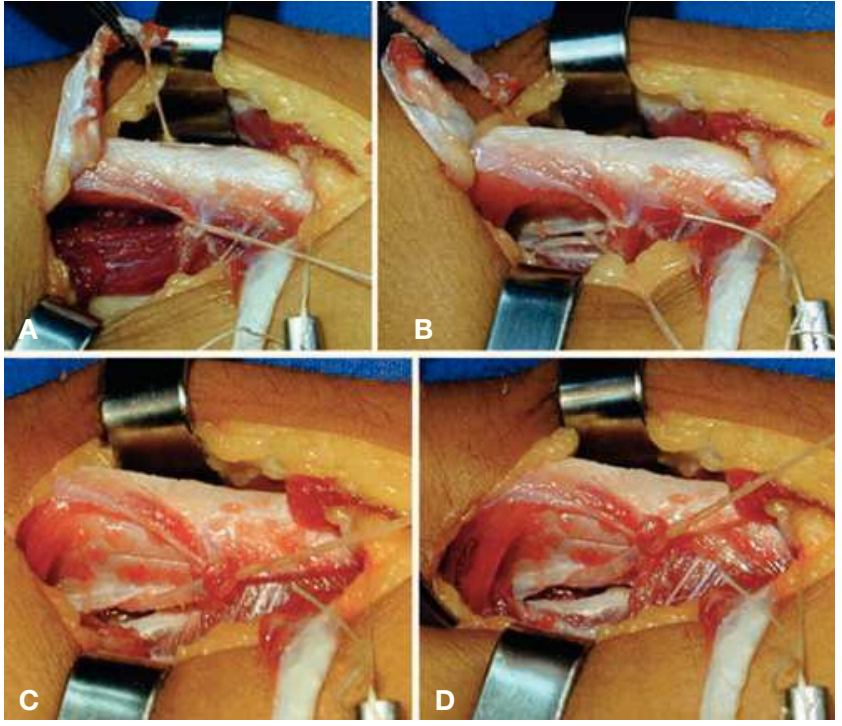

Figura 9 Transferencia del hemitendón del braquiorradial de dorsal a volar. (A y B) Paso de hilo de tracción a través de la membrana interósea y captura del hemitendón. ( $C$ y D) Paso del hemitendón del braquiorradial a través de la ventana de la membrana interósea.

que generalmente se resuelve en un período de 6 semanas.

La lesión de la arteria radial durante el procedimiento quirúrgico es posible ya que se trabaja en dicha zona durante todo el procedimiento. Realizar una hemostasia cuidadosa concluida la cirugía es muy importante para evitar
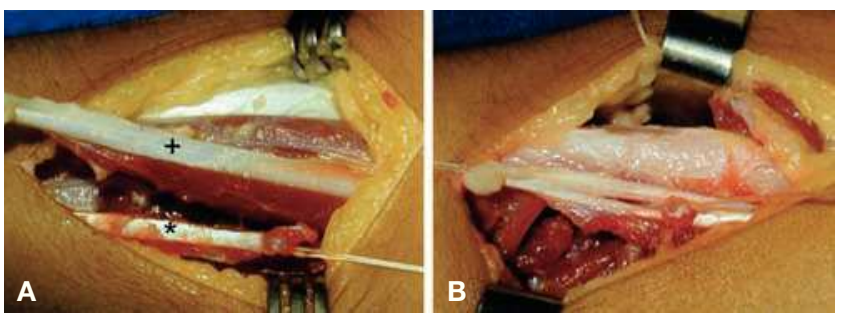

Figura 10 (A) Tendón del pronador redondo (signo suma). Hemitendón del braquiorradial (signo asterisco). (B) Sutura del tendón del pronador redondo al hemitendón del braquiorradial. 

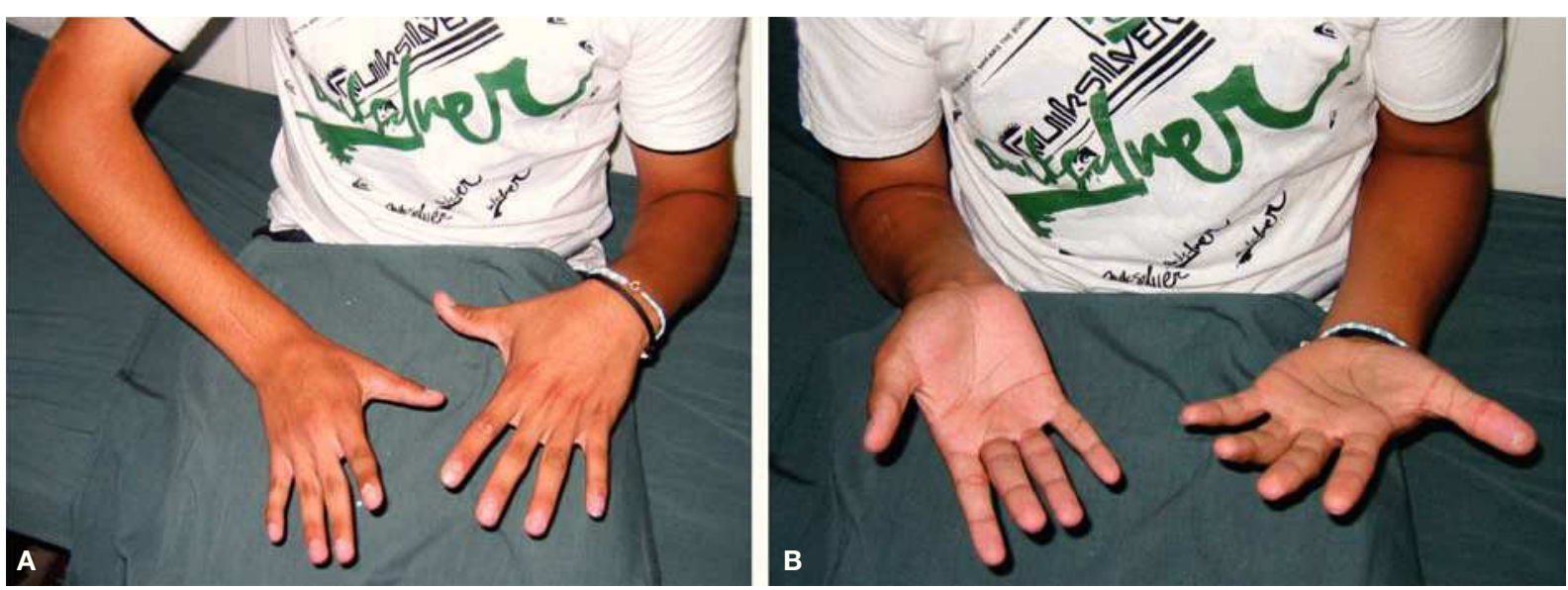

Figura 12 Octavo año postoperatorio. (A) Pronación activa. (B) Supinación activa
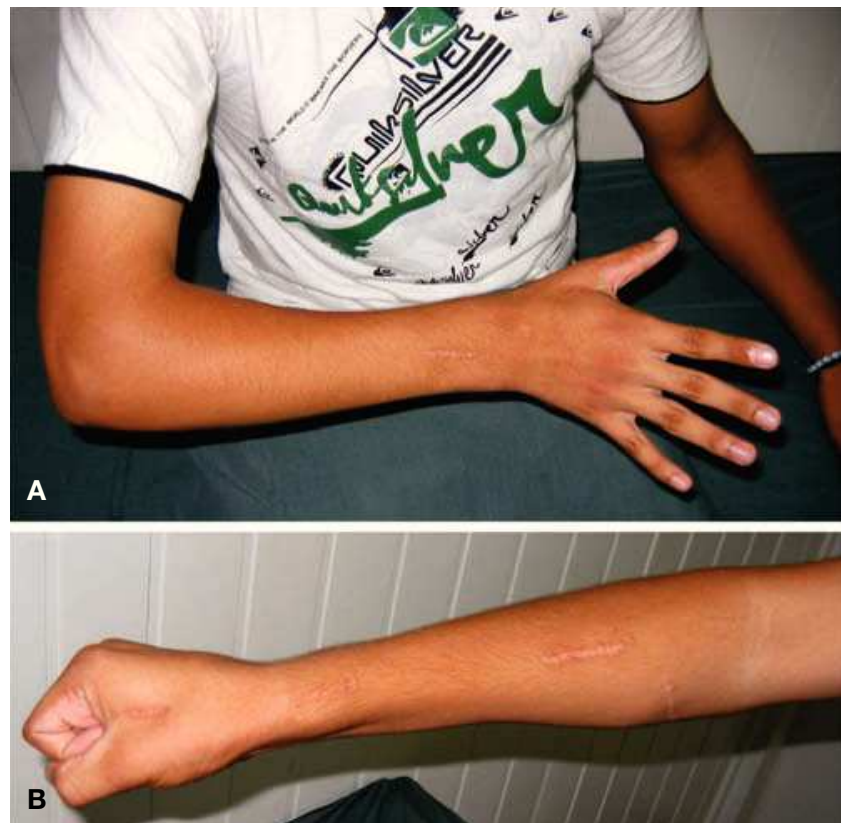

Figura 11 Octavo año postoperatorio. (A) Apertura de la mano con la muñeca en extensión y extensión del pulgar. (B) Cierre de puño completo con la muñeca en posición neutra.

hematomas y posteriores complicaciones de la herida quirúrgica.

Una posible complicación es la adherencia de la transferencia tendinosa en su paso por la ventana de la membrana interósea, que se evita no lesionando la inserción ósea de la membrana interósea.

\section{Caso clínico}

Paciente de 7 años de edad, diestro, que presentaba una hemiparesia espástica, con cociente intelectual disminuido, marcada pronación del antebrazo, pulgar en la palma y dificultad para la extensión de los dedos con la muñeca en ex- tensión pasiva. Tenía dificultad para coger y manipular objetos y realizaba el apoyo de los objetos con el borde radial del dorso de la mano y muñeca.

Se realizó la transferencia sin complicaciones y concluido el periodo de inmovilización post-operatorio se remitió a terapia ocupacional para comenzar con el protocolo rehabilitador.

Al sexto mes postoperatorio, el paciente presentaba una buena evolución clínica, con una supinación de $70^{\circ}$, flexoextensión completa de todos los dedos con extensión de muñeca y buena apertura de la primera comisura

A los ocho años de la intervención quirúrgica el paciente sigue manteniendo el movimiento de supinación (figs. 11 y 12).

\section{Conflicto de intereses}

Los autores declaran no tener ningún conflicto de intereses.

\section{Bibliografía}

1. Campbell WC, Canale ST, Beaty JH. Campbell's operative orthopaedics. 12. ${ }^{\text {a }}$ ed. Philadelphia: Mosby, Elsevier; 2013.

2. Gschwind C, Tonkin M. Surgery for cerebral palsy: Part 1. Classification and operative procedures for pronation deformity. J Hand Surg [Br]. 1992;17(4):391-5.

3. Van Heest AE, House JH, Cariello C. Upper extremity surgical treatment of cerebral palsy. J Hand Surg Am. 1999;24:323-30.

4. Bax M, Goldstein M, Rosenbaum P, Leviton A, Paneth N, Dan B, et al.; Executive Committee for the Definition of Cerebral Palsy. Proposed definition and classification of cerebral palsy, April 2005. Dev Med Child Neurol. 2005;47:571-6.

5. Bax MC. Terminology and classification of cerebral palsy. Dev Med Child Neurol. 1964;6:295-7.

6. Organization WH. International classification of functioning, disability and health: ICF. Geneva: World Health Organization; 2001.

7. Shapiro BK. Cerebral palsy: A reconceptualization of the spectrum. J Pediatr. 2004;145(2 Suppl):S3-7.

8. CM. P., An operation for the relief of flexion-contracture in the forearm. J Bone Joint Surg Am. 1923;5:233-4. 
9. Sakellarides HT, Mital MA, Lenzi WD. Treatment of pronation contractures of the forearm in cerebral palsy by changing the insertion of the pronator radii teres. J Bone Joint Surg Am. 1981;63:645-52.

10. Bunnell S. Cirugía de la mano. Buenos Aires: Intermédica; 1967.

11. Green WT, Banks HH. Flexor carpi ulnaris transplant and its use in cerebral palsy. J Bone Joint Surg Am. 1962;44-A:1343-430.

12. Colton CL, Ransford AO, Lloyd-Roberts GC. Transposition of the tendon of pronator teres in cerebral palsy. J Bone Joint Surg Br. 1976;58:220-3.
13. Inglis $A E$, Cooper W. Release of the flexor-pronator origin for flexion deformities of the hand and wrist in spastic paralysis. A study of eighteen cases. J Bone Joint Surg Am. 1966;48:847-57.

14. Zancolli EA, Goldner LJ, Swanson AB. Surgery of the spastic hand in cerebral palsy: report of the Committee on Spastic Hand Evaluation (International Federation of Societies for Surgery of the Hand). J Hand Surg Am. 1983;8(5 Pt 2):766-72. 